\title{
LAS ARGUMENTACIONES DE ESTUDIANTES DE POLIMODAL SOBRE LA INTERACCIÓN ELÉCTRI- $\mathrm{CA}^{++1}$
}

\author{
Silvia Stipcich \\ Facultad de Ciencias Exactas \\ Universidad Nacional del Centro de la Provincia de Buenos Aires \\ Buenos Aires - Argentina
}

\section{Resumen}

En este trabajo se exploran y analizan las justificaciones que estudiantes de segundo año de polimodal emplean cuando responden preguntas sobre los modelos que explican la interacción eléctrica. Para ello se seleccionan las respuestas escritas elaboradas al contestar dos preguntas correspondientes a dos temarios de una evaluación integradora, que se aplican a un mismo curso. Las dos cuestiones se proponen el reconocimiento del modelo de campo de fuerzas más explicativo que el de acción a distancia. El análisis tiene una doble intención: por una parte, busca identificar las formas argumentativas que los estudiantes emplean durante el proceso de enseñanza y aprendizaje y por la otra, aspira a generar recomendaciones didácticas que pudieran ayudar durante ese proceso.

Palabras clave: Argumentación; procesos de enseñanza y aprendizaje; interacción eléctrica.

\footnotetext{
+ Polymodal students' argumentations about electrical interaction

* Recebido: novembro de 2007. Aceito: julho de 2008.

${ }^{1}$ Este trabajo ha sido financiado por la Agencia Nacional de Promoción Científica y Tecnológica a través del FONCyT, Contrato de Préstamo BID 1728/OC-AR, proyecto PICT-05 $\mathrm{N}^{\mathrm{o}}: 34479-234$.
} 


\begin{abstract}
This article is aimed to evaluate second year students' justifications about electrical interactions. To achieve the aim the answers of two evaluation questions about electrical interactions have been explored and analyzed. The purpose of the two items was to recognize that the forces field model is better explanatory than the distance action model. The analysis have two intentions: on the one hand, to identify students' argumentations during the teaching and learning process, and on the other hand, to generate didactic recommendations that could help during this process.
\end{abstract}

Keywords: Argumentation; teaching and learning processes; electrical interaction.

\title{
I. Introducción
}

El interés por contribuir en la formación de ciudadanos críticos, capacitados para construir juicios reflexivos y pensamientos que reconozcan que no hay un solo punto de vista, ha llevado a los estudiosos en Educación en Ciencias a incluir entre los temas que merecen ser investigados, diferentes aspectos vinculados con el proceso de comunicación en las clases de ciencias. Así se han desarrollado investigaciones sobre el tipo de lenguaje empleado, los estilos de preguntas y respuestas que profesores y estudiantes intercambian en las clases, las formas de interpretación de los enunciados de problemas, etc. (LEMKE, 1990; DRIVER et al., 1994; MORTIMER, 2000).

Más recientemente y en asociación con los modos de razonamiento que se suceden en las clases están apareciendo artículos donde el énfasis se encuentra en el modo de argumentación que se sostiene en las aulas (JIMÉNEZ ALEXANDRE; DÍAZ DE BUSTAMANTE, 2003; SARDÁ; SANMARTÍ, 2000).

Hay acuerdos entre los profesores de ciencias en el reconocimiento de las dificultades que se les presentan a los estudiantes a la hora de elaborar argumentaciones personales que sean convincentes en relación con el contenido de la disciplina y también, en la búsqueda de consensos y/ó el discernimiento en un debate académico. (PÉREZ RIFO; VEGA ALVARADO, 2003) Entre otros aspectos, se advierten inconvenientes para diferenciar entre hechos observables e inferencias, es habitual que no reconozcan términos de uso científico respecto de los de uso 
cotidiano, que no puedan justificar ninguna afirmación. (SARDÁ; SANMARTÍ, 2000).

La importancia que puede tener el desenvolver formas argumentativas para la formación de ciudadanos con capacidad para optar ante diferentes alternativas fundamentando la decisión en el conocimiento científico se incorpora como un aditamento más al objetivo, más amplio que la sola justificación, de procurar aprendizajes significativos. Es decir, en la escuela el propósito es doble: preparar a los estudiantes para desenvolverse haciendo uso de formas argumentativas a la vez que propiciar el aprendizaje significativo de los contenidos de las argumentaciones. En palabras de Lemke (1997) se hace necesario que los estudiantes dominen tanto el patrón temático, vinculado al campo disciplinar específico, como el patrón estructural, propio del tipo del texto científico que se esté solicitando.

En este trabajo se exploran y analizan las justificaciones que emplean los estudiantes de segundo año de polimodal cuando responden preguntas sobre los modelos que explican la interacción eléctrica ${ }^{2}$. El análisis tiene una doble intención: por una parte, busca identificar características en de las formas argumentativas que los estudiantes construyen y por la otra, se aspira a generar recomendaciones didácticas que, de incorporarse a la práctica docente, pudieran ayudar a los estudiantes en la elaboración de este tipo de discurso.

\section{I.1 Aprendizaje escolar y argumentación}

En las aulas de ciencias el proceso de argumentación puede entenderse como la construcción y justificación del conocimiento escolar en una disciplina particular como la Física. Se trata de un proceso que se enmarca en el paradigma constructivista. Desde este enfoque, cada individuo es el constructor de su propio conocimiento a partir de darle sentido a su aprender intentando relacionarlo con su experiencia previa.

El aprendizaje es visto como un proceso adaptativo del aprendiz en el intento por ajustar el mundo que experimenta, o que se le presenta, en función de sus experiencias previas (SÁNCHEZ ILABACA, 2004). Desde esta postura el conocimiento es construido por cada sujeto a partir de las acciones que este sujeto realiza sobre la realidad. La realidad existe en tanto existe una estructura mental interna del aprendiz, capaz de interpretarla. Diferentes autores hablarán de diferentes estructuras internas en los sujetos, tales como modelos mentales, esquemas, esque-

${ }^{2}$ La justificación es apenas uno de los elementos de un proceso argumentativo. 
mas de asimilación, etc. que le permitirán al sujeto interactuar con esa realidad. El énfasis está dado en la forma en que los sujetos construyen conocimiento a partir de ideas, creencias y saberes previos (MATURANA, 1995; NOVAK, 1998). El conocimiento no es una copia de la realidad, es una construcción personal de la realidad.

Algunos teóricos dirán que el conocimiento que se construye es situado y es parte y producto de la actividad, el contexto y la cultura donde se desarrolla y se lo utiliza. Los seguidores de esta postura otorgan especial importancia a las actividades de aprendizaje, y al ámbito o las condiciones en que se las desenvuelve. El aprendizaje puede entenderse también, como un proceso por el cual los aprendices se van apropiando paulatinamente de la cultura y las prácticas sociales de una comunidad. (DÍAZ BARRIGA, 2003)

En términos vigotskianos, el aprendizaje consiste en la internalización de los símbolos, signos y prácticas de la ciencia. Es claro que para acceder a este proceso de enculturación, como suele denominarse, se precisa de la intervención de sujetos más experimentados. En el caso del aprendizaje escolar, es el docente el necesario nexo para propiciar espacios de mediación y negociación de significados ajustando las actividades de enseñanza a las necesidades del grupo escolar que se trate.

Las palabras no contienen el significado en sí mismas, están basadas en las construcciones que los sujetos hacen con ellas. El significado es una construcción personal. Lakoff (1987) sostiene que el significado de un objeto (palabra, instrumento) está "corporeizado" a partir de aquello que podemos hacer con ese objeto.

Que el significado de las palabras es algo que está en las personas que emplean esas palabras quiere decir que no se trata de un ingrediente que forma parte de la palabra. Por el contrario, el significado es un resultado del proceso de interacción entre la persona y la palabra. El significado es relativo (LERNER, 1994). Es decir, cada persona atribuye un significado a las palabras que depende de su desarrollo cognitivo, de sus experiencias previas, de su situación emocional, etc.

Esta aproximación a la noción del significado de un determinado objeto, concibe a la acción como génesis del conocimiento de ese objeto. Se trata de una acción funcional, dirigida hacia un objetivo dentro de una cierta cultura. Es decir, lo que motoriza a la acción es el uso social del objeto que se quiere construir (PERINAT, 1995). 
La comunicación es posible porque asignamos significados que son compatibles a los significados que otros miembros de nuestra comunidad asignan. En los casos que no son coincidentes es necesario reconstruirlos. Este proceso es fundamental para la comunicación, es donde la cooperación entre aprendices y docente y aprendices entre sí, cobra especial relevancia. Los "otros" de la comunidad a la que pertenecemos, son quienes nos posibilitan la oportunidad de contrastar nuestro mundo de experiencias. En esa contrastación hacemos adaptaciones para ensamblar nuevos conocimientos a las experiencias que ya tenemos (MATURANA, 1995).

La mayor parte de la actividad áulica se desenvuelve en alguna forma de lenguaje. Esto justifica, en buena medida, el lugar preponderante que los estudios sobre lenguaje escolar y los diversos géneros discursivos vienen cobrando en los últimos años (SUTTON, 1997; LEMKE, 1997).

Cuando las clases de ciencia son entendidas como espacios de opiniones divergentes es de particular importancia que los alumnos elaboren respuestas razonadas produciendo argumentos que puedan ser analizados, revisados y negociados con miras a construir conocimiento compartido acerca del mundo físico. En particular, se aspira a promover modos de producir textos argumentativos que faculten a los estudiantes en la toma de decisiones desde posiciones científicamente aceptables. Es decir, se espera que puedan apoyar una opinión con pruebas o razones. Este propósito se enmarca en los lineamientos pragmáticos que conciben a la argumentación como un conjunto de estrategias discursivas dirigidas a la demostración de una opinión (PÉREZ RIFO; VEGA ALVARADO, 2003).

Para argumentar hay que expresarse, es decir, construir su punto de vista, y dar razones que lo respalden, que lo apoyen; interactuar, es decir, escuchar a los otros, integrar lo que han dicho de su propio discurso, y así, combatir el ensimismamiento; y, también, dudar, vivir la experiencia de la incertidumbre, lo que ocurre cuando parece que los discursos de los antagonistas se equilibran (PLANTIN, 2004, p. 124).

La argumentación es una actividad de naturaleza discursiva que se realiza justificando tanto los puntos de vista sobre un tema como sus objeciones con el propósito de que las posiciones defendidas se vuelvan aceptables para quienes va dirigida la argumentación. En este sentido el discurso argumentativo se convierte en una forma de regulación del pensamiento que posibilita al individuo a reflexionar sobre los fundamentos y los límites de las propias perspectivas (LEITAO, 2003). 
Esta postura se enrola en las filas de la pragmática de la argumentación o pragmadialéctica (VAN EEMEREN; GROOTENDORST, 2002) donde se aspira a dar solución a una diferencia de opinión, partiendo del argumento como una clase de interacción, que surge en un contexto determinado dentro del discurso. El aspecto dialéctico se asocia a que en la discusión participan dos partes (incluyendo el discurso monológico) ${ }^{3}$. El aspecto pragmático atiende a la incorporación de conocimientos que amplían el análisis de los argumentos, avanzando mucho más allá de lo que lo hacen los enfoques puramente lógicos incorporando elementos del contexto en que se sitúa el debate.

... el lenguaje opera en situaciones humanas y no mediante proposiciones desituadas y separadas de las estructuras más amplias de la vida humana (TOULMIN 2003, p. 29)

La posibilidad de producir argumentaciones adecuadas requiere el desenvolvimiento de habilidades comprensivas y productivas estrechamente vinculadas con la situación en la que un sujeto se encuentra involucrado. Esta característica es la que impide la determinación de una secuencia estricta para los diversos tipos y géneros de discurso.

La adquisición de la competencia argumentativa se ubica como un tipo textual de adquisición tardía, propio de los estadios de pensamiento más formales. Si bien es cierto que desde muy niños, los sujetos aprenden a justificar sus conductas, desde el punto de vista discursivo textual se trata apenas de protoargumentaciones que combinan conductas verbales y no verbales tales como la insistencia, la repetición, el contacto físico, el llanto. (SILVESTRI, 1997).

El aprendizaje de la argumentación amplia las habilidades comunicativas a la vez que, como otras formas discursivas, traduce procedimientos conceptuales de los sujetos (WERTSCH, 1993). La argumentación es una forma particular de discurso que surge de un motivo o necesidad, que determina acciones concretas para responder a una intencionalidad. Esta intencionalidad demanda operaciones cognitivas ubicadas en una determinada situación sociocultural permitiendo no sólo la comunicación con el mundo, sino también la transformación y recreación de la realidad natural y social.

${ }^{3}$ El discurso monológico es aquél en el que participa un solo interlocutor aunque puede conformar un discurso de dos partes entre él consigo mismo o bien, él y el texto que se le presenta. 
Para poder argumentar se requiere de la regulación de procesos tales como la abstracción, la generalización y la particularización según las demandas de la situación presentada para el debate. Es decir, se necesita resituar los conceptos aprendidos y formulados en un cierto nivel de abstracción hacia otros niveles diferentes $^{4}$. Se trata de una operación muy compleja que solo llega a dominarse hacia finales de la adolescencia (VIGOTSKY, 1963).

\section{I.2 Estructura de un texto argumentativo}

La estructura de un texto argumentativo se compone de dos aspectos fundamentales (PÉREZ RIFO Y ALVARADO, 2003)

a) El marco pragmático: incluye el universo de conocimiento que comparten o disputan los sujetos argumentadores (patrón temático en términos de Lemke) y el universo discursivo en cual se produce la argumentación.

b) El componente sintáctico textual: incluye a las categorías funcionales de una argumentación.

La estructura del modelo argumentativo que propone Toulmin (1958) contiene, básicamente, tres elementos o categorías fundamentales y otros tres opcionales.

Tesis o opinión: es la idea o afirmación que se quiere probar, aquella sobre la que se reflexiona.

Conclusión o argumento: es el conjunto de hechos, palabras o datos, cadenas racionales que el argumentador transforma en razones para apoyar la tesis y presentarlos como prueba. Muchas veces, desde los argumentos es posible inferir la tesis.

Garantía o justificación: es una premisa, norma, ley o regla general que justifica la validez de la argumentación. Es un axioma que no se discute porque se supone consensuado entre los interlocutores, razón por la cual suele estar implícita.

Estos tres primeros elementos son los determinantes de un texto argumentativo.

Opcionalmente, de manera implícita o explícita, pueden considerarse a los siguientes otros tres componentes:

\footnotetext{
${ }^{4}$ En adhesión a Toulmin (2003) que propone enunciados situados o desituados, se alude aquí a la posibilidad de volver a situar (resituar) un concepto ya empleado en una situación diferente.
} 
Fuente: es una proposición que respalda y sirve como medio de prueba de la tesis o la verdad de los datos.

Calificador: es un enunciado que relativiza la tesis o los argumentos que se han propuesto. Suele aparecer en forma de conectores del tipo: "creo que..." "algunas veces..."

Reserva: es una proposición que limita el alcance de la tesis ya que la misma no tiene un carácter absoluto.

En síntesis el modelo de Toulmin puede representarse de la siguiente forma:

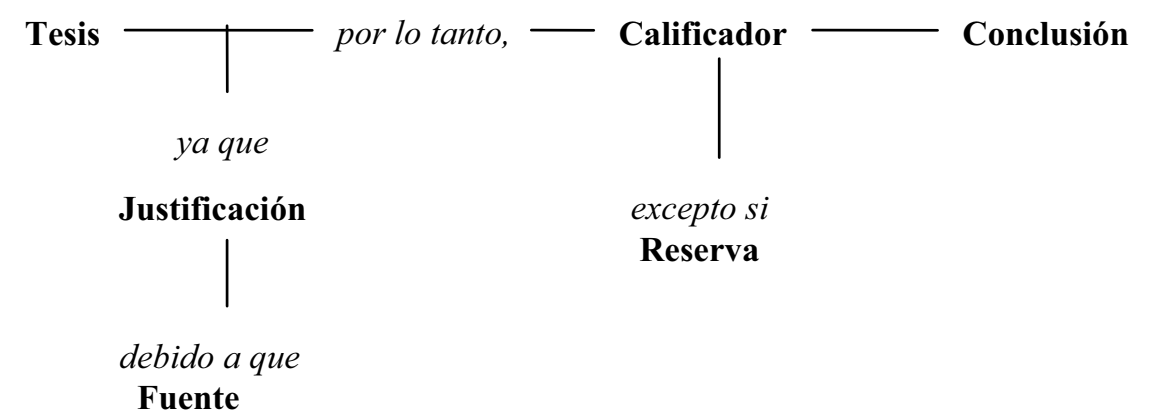

Fig. 1 - Elementos del modelo argumentativo de Toulmin.

Como puede apreciarse en la Fig. 1 la estructura de un texto argumentativo, según el modelo de Toulmin, adopta la forma de un movimiento que avanza desde la tesis a la conclusión, pasando por la justificación. Este esquema secuencial es también compartido con el proceso de demostración que se desplaza desde la premisa (hipótesis) a la conclusión. La diferencia estriba en que mientras que la demostración se ocupa de la relación necesaria entre hipótesis y conclusión, la argumentación utiliza con más frecuencia las relaciones de probabilidad, credibilidad y posibilidad.

La estructura representada en la Figura 1 está formada por enunciados que se enlazan entre sí, de manera que uno de ellos, denominado conclusión, aparece como resultado de los demás, llamados premisas de la argumentación. Esta argumentación es la expresión lingüística de una serie de actividades cognitivas desarrolladas por el sujeto. Esas representaciones que se plasman en la manifestación escrita son indicadores del tipo de razonamiento que el sujeto ha desarrollado. Aún 
cuando los procesos argumentativos que se suceden en el aula no responden a razonamientos en los términos que los entiende la lógica formal, es deseable, a los fines de una adecuada argumentación, la coherencia de las justificaciones en relación con la tesis y la conclusión a la vez que su aceptabilidad desde el referente disciplinar de la Física.

Las premisas pueden ser entendidas como los motivos o hechos por los cuales debería aceptarse la conclusión.

En ese proceso de desarrollar una justificación o relacionar premisas para arribar a una conclusión, los sujetos ponen en funcionamiento diversas formas de razonamiento como el razonamiento por causas, la inducción, la deducción. Es decir, conforman un entramado de procesos cognitivos que sirven de base o sustento a las argumentaciones.

Es habitual que en los procesos discursivos donde se pretende debatir una opinión aparezcan razones que no atienden a la opinión sino a atacar o desacreditar a la persona que emite esa opinión. En ese caso, se dice que se argumenta de manera falaz.

Las falacias son entendidas como falsas argumentaciones en el sentido de que parecen responder a la estructura de una argumentación válida, es decir, parecen válidas pero infringen las reglas del buen acto argumentativo. Algunas falacias se relacionan con la ambigüedad o incomprensibilidad de los enunciados, la ausencia de significados, etc. Otras, se encuentran asociadas al rol de los sujetos que emiten la argumentación, tales como insultarlo, obligarlo a aceptar tesis que son inconsistentes, etc. (PÉREZ RIFO Y VEGA ALVARADO, 2003)

Todas las falacias son razonamientos que vulneran alguna regla lógica. Van Eemeren y Grootendorst (2002) proponen una tipología de falacias tomando como criterio qué tipo de infracción contiene la falacia respecto a las reglas básicas que debe cumplir toda buena argumentación. Las diez reglas que toda buena argumentación debe cumplir, son las siguientes:

1) Los involucrados en la disputa no deben crearse impedimentos.

2) Si alguien expresa una opinión debe disponerse a defenderla, si se lo piden.

3) Cuando se ataca una argumentación hay que centrarse en la tesis que se ha anunciado, sin desviar el discurso, sin presentar la tesis de forma diferente.

4) Los argumentos que defienden a una tesis deben estar relacionados con ella y no con otra tesis. 
5) Toda persona debe aceptar las consecuencias y la existencia de las premisas que deja implícitas y, en consecuencia, debe aceptar que se le ataque en ese terreno.

6) Una tesis puede considerarse defendida si se basa en argumentos pertenecientes a un punto de partida común.

7) Una tesis puede considerarse defendida de forma adecuada si la defensa se desarrolla con el uso de argumentos que reflejen y respeten la praxis y el esquema argumentativo comúnmente aceptados.

8) Los argumentos usados en una discusión deben ser o haberse vuelto válidos, haciendo explícitas algunas de las premisas que quedaban implícitas.

9) Una defensa que pierde debe tener como consecuencia que el sujeto argumentante acepte cambiar su posición, mientras que una defensa vencedora debe tener por consecuencia que el antagonista cambie su posición y retire las dudas sobre la tesis defendida por el sujeto argumentante.

10) La formulación de las tesis, de las posiciones recíprocas y de los argumentos debe ser lo más clara y comprensible posible.

Como puede advertirse del listado anterior, algunas de las reglas se asocian al rol de los sujetos que participan del debate mientras que otras se vinculan con los elementos constitutivos de la estructura argumentativa en sí misma. Estas últimas son las de importancia para esta presentación, siendo que se ocupa de producciones escritas y no del debate oral.

\section{Participantes, situación y registro de los datos}

Los datos que se analizan en esta presentación han sido extraídos de un corpus más amplio que pertenece a una investigación sobre la conceptualización de la interacción electromagnética entre estudiantes de segundo año de polimodal de la escuela Nacional Ernesto Sábato de la ciudad de Tandil (dependiente de la Universidad Nacional del Centro de la Provincia de Buenos Aires).

Estos estudiantes cursan Física por segundo año consecutivo dentro de la institución en la que se desenvuelve este trabajo. Las clases de Física son concebidas como espacios de negociación de significados con énfasis en el intercambio de opiniones entre los alumnos entre sí y de los alumnos con el docente. Para propiciar el debate el docente plantea a lo largo de cada clase cuestiones que vehiculizan la posibilidad de responderse por opiniones divergentes. La intención de esta modalidad de trabajo es la de "provocar" el debate y con él, posibles argumentaciones procurando favorecer la emergencia, revisión y transformación de perspectivas 
respecto del mundo físico. La dinámica de trabajo enfatiza las capacidades que se aspira que los estudiantes plasmen en sus escritos tales como: proveer de justificaciones a los enunciados que se pronuncian, ofrecer argumentaciones y refutaciones, según sea el caso y brindar argumentos alternativos para una misma opinión.

Una clase típica comienza con el tratamiento de un tema específico que es presentado como un problema.

Los problemas tienen un poder especial para dar vida a una historia de la creación de nuevas ideas o del descubrimiento de nuevos hechos; $y$ es que son el origen de la lucha del cientifico por crear una nueva teoría o hacer un nuevo experimento. Si uno entiende el problema con que se enfrentaba el cientifico, se puede participar de alguna forma en su proceso de pensamiento, en su intento de solución y en su posible descubrimiento (BERKSON, 1981, p. 30).

Mediante una discusión oral se procura el desenvolvimiento de respuestas alternativas que aspiren a constituirse en posibles soluciones del problema. Los estudiantes cuentan con un material de apoyo, producido por el investigador. Se trata de un material teórico-práctico ya que integra desarrollos teóricos con actividades especialmente diseñadas. Otro material de estudio de los alumnos son las carpetas de trabajo donde registran (mediante la toma de apuntes) las interpretaciones de lo acordado en los debates áulicos, también capítulos de determinados textos oportunamente seleccionados por el docente.

Un conjunto de estudios previos facilitaron el reconocimiento del nivel de conceptualización de los estudiantes en cuestión. Esto permitió la elaboración del material a partir de la definición de un conjunto de invariantes operacionales que se aspira a desenvolver entre los alumnos con miras a acortar la brecha entre los conocimientos que detentan y los científicamente consensuados (STIPCICH, 2006).

El enfoque de enseñanza empleado durante la investigación es el es que Pozo y Gómez Crespo, (1998) denominan por explicación y contrastación de modelos. Se trata de una visión donde el alumno reconstruye los métodos, valores y sistemas conceptuales científicos mediados con la ayuda del docente, quien colabora para que los conocimientos se vuelvan comprensibles y contrastables. El núcleo organizador de este enfoque son los diferentes modelos con que se representa el conocimiento en un dominio específico. Se asume que el profesor es capaz de crear ciertos escenarios explicativos para hacer dialogar a los estudiantes acerca de los fenómenos que se estudian. En este caso, se espera que las situaciones que 
se generan en el aula colaboren para que los estudiantes sean capaces de explicar la interacción eléctrica a partir del empleo de los modelos de acción a distancia y campo.

Para esta investigación, los procesos argumentativos se constituyen en indicadores del domino que los estudiantes tienen tanto del patrón temático (los contenidos involucrados) cuanto del patrón estructural (la forma discursiva empleada) del texto que elaboran.

Se recogen las producciones escritas cuando resuelven trabajos prácticos de lápiz y papel y de laboratorio a la vez que las producciones individuales en caso de situaciones de evaluación. Asimismo se registran en audio las discusiones grupales que enmarcan a cada una de las clases durante las cuales se abordan los bloques temáticos energía eléctrica y circuitos eléctricos.

Para esta presentación se seleccionan las respuestas escritas elaboradas al contestar dos preguntas correspondientes a dos temarios de una evaluación integradora, que se aplican a un mismo curso. La misma se lleva a cabo al finalizar el tratamiento de los temas del primer cuatrimestre de la cursada de Física de segundo año polimodal del año 2006.

La particularidad de las dos cuestiones es que ambas se proponen el reconocimiento del modelo de campo de fuerzas como superador del modelo de acción a distancia o bien, dicho de otra manera, se aspira a superar la primera aproximación que consiste en describir el comportamiento de las cargas por medio de las fuerzas que actúan sobre ellas.

Las dos preguntas sobre las informa esta presentación son las siguientes:

Tema 1: Comenta qué diferencia hay entre la noción de campo de fuerzas y la de fuerza eléctrica.

Tema 2: ¿Qué importancia tiene la introducción de la noción de campo eléctrico para describir la interacción entre cargas?

Lo que se está solicitando a los estudiantes es, en los dos casos, la identificación de la "novedad" que introduce el campo que es el reemplazo de la idea de una acción a distancia, encarnada en una fuerza aplicada a cada carga, por la presencia de un campo que se constituye en un mediador para esa interacción.

Cualquiera de las preguntas formuladas permiten inferir (haciendo uso de los elementos del modelo de Toulmin) sendas conclusiones. La pregunta del tema 1 contiene la afirmación de que el campo de fuerzas y la fuerza son cosas diferentes, la del tema 2, sostiene que la introducción de la noción de campo eléctrico es importante para describir la interacción entre cargas. 
Un esquema argumentativo básico (Tesis, justificación y conclusión) para cada una de las preguntas propuestas podría ser el siguiente:

La interacción eléctrica puede explicarse por los campos que generan las por lo tanto, El campo de fuerzas cargas o por la fuerza entre ellas y la fuerza son dos<smiles>CCCCCC</smiles>

\section{ya que}

se puede pensar en una interacción mediada por el espacio que separa a las cargas o por una acción a distancia

Fig. 2 - Una posible estructura argumentativa para la pregunta del tema 1.

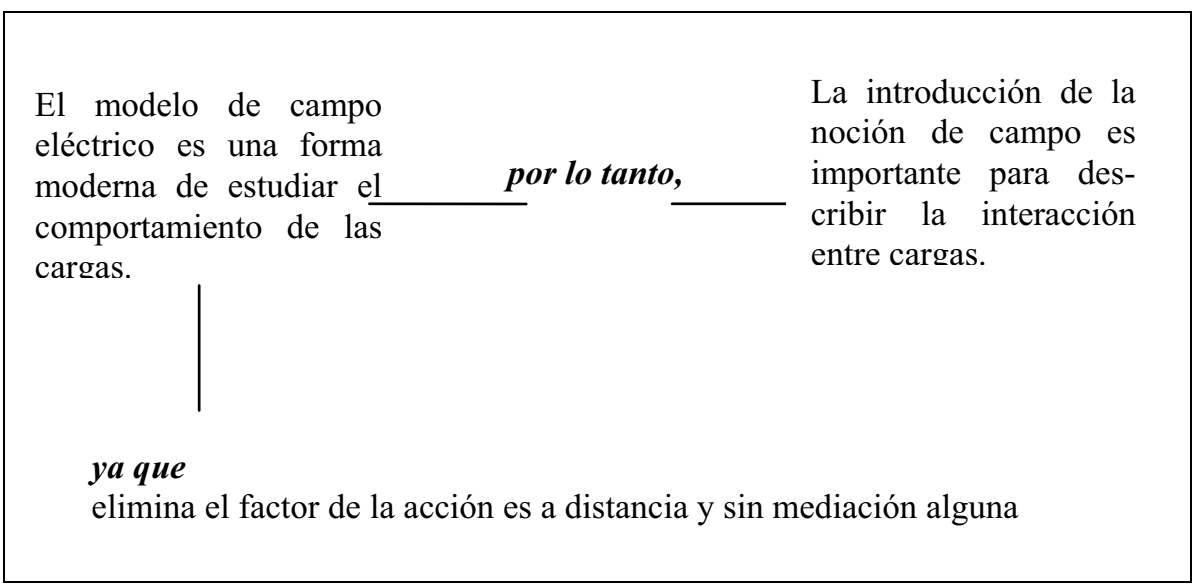

Fig. 3 - Una posible estructura argumentativa para la pregunta del tema 2. 
En cualquiera de los dos casos, se espera que las respuestas puedan listar características de cada modelo. Los estudiantes suelen referirse a la importancia de un determinado fenómeno a partir de la enumeración de las características que lo destacan. En este caso, la eliminación de la idea de acción a distancia es el fundamento por excelencia que los estudiantes podrían presentar para destacar la introducción de la noción de campo, por ejemplo. Sin embargo, hay otras proposiciones que podrían formar parte de las justificaciones, tanto para avalar la importancia de incorporar al modelo de campo de fuerzas, como para establecer diferencias respecto de la concepción de una interacción a distancia, tales como

\begin{tabular}{|l|l|}
\hline \multicolumn{1}{|c|}{ Modelo de acción a distancia } & \multicolumn{1}{c|}{ Modelo de campo de fuerzas } \\
\hline Interactúan las cargas & Interactúan los campos \\
\hline $\begin{array}{l}\text { La fuerza actúa en cada una de las } \\
\text { cargas que participan de la interacción }\end{array}$ & $\begin{array}{l}\text { La fuerza se define en cada punto del } \\
\text { espacio }\end{array}$ \\
\hline $\begin{array}{l}\text { La presencia de la fuerza es simultánea } \\
\text { (el tiempo de propagación es cero) }\end{array}$ & $\begin{array}{l}\text { Transcurre un tiempo hasta que la } \\
\text { fuerza se percibe (se propaga punto a } \\
\text { punto) }\end{array}$ \\
\hline Vale para cargas en reposo & $\begin{array}{l}\text { Es válido con independencia de que } \\
\text { las cargas estén o no en reposo }\end{array}$ \\
\hline $\begin{array}{l}\text { Necesita al menos de la presencia de } \\
\text { dos cargas }\end{array}$ & $\begin{array}{l}\text { No necesita de dos cargas, cada carga } \\
\text { genera un campo }\end{array}$ \\
\hline
\end{tabular}

Fig. 4 - Diferencias entre los modelos de acción a distancia y campo de fuerzas para el caso de cargas eléctricas.

Para dar cuenta de los resultados se procede a la trascripción textual de todas y cada una de las respuestas y a la lectura reiterada de las mismas con el propósito de identificar regularidades entre algunas respuestas y conformar las categorías que se comentan en el apartado que sigue.

\section{Resultados}

De un total de 23 respuestas, 13 corresponden al tema 1 y 10 al tema 2 .

El análisis llevado a cabo contempla dos instancias:

a) evaluación de la coherencia o concordancia entre la justificación, la tesis y la conclusión. En ocasiones los estudiantes aluden, en sus respuestas, a leyes 
o a principios de la Física que nada tienen que ver con los enunciados presentados. Sin embargo, el carácter de autoridad científica suele ser un lugar común en las respuestas. Esta evaluación se propone, antes de analizar la calidad de los argumentos, detectar si las construcciones elaboradas son pertinentes en relación con la tesis que se enuncia y la garantía o justificación a la que se recurre. Este aspecto pone el énfasis en del patrón estructural;

b) calidad de las justificaciones o garantías. Se espera discriminar a las justificaciones según los niveles de explicitación que los estudiantes desenvuelvan en sus respuestas. La explicitación alude a los conceptos y procesos físicos que emplean en las respuestas y al lugar que los mismos ocupan dentro de la producción del texto escrito, es un indicador de la validez científica que las mismas presentan según el grado de acuerdo que tienen con los contenidos científicos. Aquí el acento está puesto en el patrón temático.

Si bien los enunciados de las preguntas formuladas no contienen en sí mismos a la tesis, ésta puede inferirse a partir de la interpretación de la afirmación que se hace. Por su parte, las justificaciones o garantías permitirán, al profesor, el reconocimiento de los sustentos disciplinares que los estudiantes pueden poner en juego frente a las preguntas formuladas.

\section{III.1 Evaluación de la concordancia entre la tesis, la conclusión y la justifica- ción}

Se admite que hay coherencia entre los tres elementos principales del modelo de Toulmin cuando, en el caso del tema 1 los estudiantes son capaces de reconocer que es necesario presentar (por enumeración o explicación) aspectos diferenciadores de una y otra noción. Para el caso del tema 2, se aceptará que una respuesta es coherente si se identifican motivos que ponen de relieve que hay una característica distintiva en la noción de campo respecto de otras nociones.

Según Lo Cascio (1998) existen, al interior de un texto argumentativo, ciertos elementos que él denomina indicadores de fuerza cuyo comportamiento permite organizar textualmente un acto argumentativo. Algunos indicadores ordenan el discurso (primero, luego, por un lado, etc.); otros son simples operadores (más, casi, sólo, apenas, etc.) y otros son conectores que expresan relaciones y orientan la interpretación de lo que se enuncia, (causa, consecuencia, oposición, etc.). En ocasiones pueden emplearse como indicadores de fuerza la reiteración de las propias palabras del enunciado de la pregunta. 
En el caso de las preguntas propuestas para esta comunicación, son los indicadores de fuerza, los que permiten decidir si la producción escrita comporta coherencia entre los elementos constitutivos.

Respuestas coherentes: se consideran aquí a aquellas producciones que, haciendo uso de determinados conectores (indicadores de fuerza), elaboran un texto que explicita lo solicitado.

- Para el tema 1 se consignan, dentro de esta categoría 9 respuestas. Ejemplos de ella son los siguientes :

Respuesta alumno: Las diferencias que existen entre campo y fuerza eléctrica son que el campo es una propiedad del espacio y que se genera por una carga que se expande indefinidamente y que no necesita de otra carga para "estar" mientras que la fuerza eléctrica se pone en evidencia cuando otra carga aparece en el campo eléctrico (modelo de campo) o cuando una carga interactúa con otra (modelo de acción a distancia).

Respuesta alumno: Campo eléctrico es la condición que genera en el espacio la partícula cargada, en cambio, fuerza es el efecto que sufre una segunda carga al estar colocada en el campo eléctrico de otra partícula eléctrica.

Los ejemplos seleccionados reflejan a los tres indicadores que los estudiantes han empleado: las propias palabras del enunciado de la consigna: las diferencias, mientras que y en cambio. En cualquiera de los tres casos su presencia pone de manifiesto la intención del estudiante por presentar una respuesta acorde a lo que solicita la pregunta enunciada.

- Para el tema 2 se registran 8 respuestas en esta categoría. Ejemplos de ella son los siguientes:

Respuesta alumno: La importancia de éste para describir el comportamiento de las cargas eléctricas es que, en las cargas eléctricas las cargas con distinto signo se atraen y las que tienen igual signo se repelen.

Respuesta alumno: Es bueno introducir la noción de campo para entender el comportamiento de una particula cargada. Hay mucha relación entre estos

${ }^{5}$ Las palabras subrayadas son los indicadores de fuerza. 
En este caso, el indicador es bueno aparece como sinónimo de la importancia asignada a la noción de campo.

Hay que notar que la coherencia entre los elementos básicos de un texto argumentativo no es garantía de una respuesta correcta desde los cánones científicamente consensuados.

Respuestas no coherentes: se incluyen producciones que no incorporan conectores durante la redacción de manera que corre por cuenta del lector inferir lo solicitado.

- Algunos ejemplos para el tema 1:

Respuesta alumno: Campo eléctrico: toda carga en el espacio lo modifica en todos los puntos. El campo eléctrico es infinito.

Fuerza eléctrica es la fuerza de interacción entre dos cargas (de atracción o de repulsión), con sentido, magnitud y la misma intensidad.

Respuesta alumno: $E$, con una carga alcanza. $F$, se piensa en el modelo de acción a distancia. El campo eléctrico es una propiedad del espacio generado por una carga eléctrica que provoca la experimentación de una fuerza eléctrica de otra carga colocada. En pocas palabras, el campo eléctrico es uno de los modelos que explica cómo es la interacción entre cargas, es decir, cómo se lleva a cabo una fuerza entre cargas.

El primero de los ejemplos seleccionados se limita a presentar una especie de definición para cada uno de los conceptos que se aspira a que se diferencien. La diferenciación debería, en este caso, realizarse por cuenta del lector.

En la segunda trascripción se comienza por caracterizar a qué refiere cada concepto. Finalmente se advierten dificultades para establecer la diferenciación campo/fuerza:"... el campo eléctrico es uno de los modelos que explica cómo es la interacción entre cargas, es decir, cómo se lleva a cabo una fuerza entre cargas".

- Ejemplos para el tema 2:

Respuesta alumno: Al tener una noción sobre lo que es un campo eléctrico sabemos que al colocar una partícula cargada en el espacio esta creará una condición en el espacio. El comportamiento variará según las otras cargas que se agreguen a su campo eléctrico. Si estas cargas son del mismo signo se alejarán, pero si son de distinto signo se pegarán. Al tener ya más de dos cargas el comportamiento variará según el signo y carga de cada una. La fuerza total que cada carga recibe es siempre la resultante de todas las fuerzas que actúan sobre ella. 
Respuesta alumno: El campo eléctrico será el espacio que una partícula cargada adoptará. Para luego comprender el comportamiento de esta carga deberíamos introducir a este campo generado anteriormente una segunda carga (carga de prueba). El valor del campo actuará directamente en el valor de la fuerza originada por la interacción de la carga. De modo que si no existiese el campo propio de cada partícula cargada no podríamos analizar el comportamiento que se originaría en relación con la otra carga.

Las respuestas anteriores son narrativas que contienen, en algunos casos, proposiciones científicamente aceptables. Sin embargo, no se refieren a lo que se pregunta directamente, quedando a cargo del lector la inferencia de la posible respuesta.

Así, en el primero de los ejemplos anteriores, podría inferirse que la fuerza se presenta como un concepto que se encuentra "subsumido" o que aparece "a consecuencia" de la presencia de un campo. Pero no es algo que se diga explícitamente.

En la segunda trascripción podría pensarse que la importancia está en que la noción de campo permite comprender el comportamiento de las partículas cargadas. No obstante, la respuesta no permite reconocer motivos que hagan aparecer al campo como más importante que otros modos para comprender el comportamiento de las cargas.

\section{2 Evaluación del tipo de justificación empleada}

En relación con el tipo de justificaciones se han conformado las dos categorías básicas (se explicitan o no se explicitan las justificaciones) y, dentro de ellas, algunas subcategorías vinculadas al contenido involucrado. Las mismas se comentan a continuación con ejemplos para cada una de ellas.

1. La justificación es explícita y aceptable: Se consignan aquellas respuestas en las que es posible reconocer un justificativo acorde al patrón científicamente consensuado.

- En el caso del tema 1, se registran 5 respuestas. Entre otras:

Respuesta alumno: El campo eléctrico es lo que genera una carga en el espacio, en cambio fuerza es lo que ejerce una carga sobre otra. Es decir, la fuerza eléctrica es una interacción que se da dentro de un campo. 
Es de destacar que el autor de esta respuesta ha asumido al modelo de campo como más explicativo que el de acción a distancia ya que habla de la fuerza a partir de la mediación del campo aludiendo a que "...se da dentro de un campo"

te:

- Para el tema 2 se consignan 2 respuestas. Ejemplo de ellas es la siguien-

Respuesta alumno: Es importante porque lo que antes era nada, ahora es campo eléctrico. Es más lógico que dos cargas se atraigan o se rechacen porque interactúan con sus campos que porque no haya nada. La noción de campo sirve más para cargas en movimiento, el modelo de acción a distancia no. El campo llena el espacio que hay entre las cargas y ya no es a distancia. Además no se necesitan dos cargas.

En esta repuesta se explicitan varias razones que privilegian a la noción de campo. Destaca tanto su función de completar el espacio entre las cargas (el alumno se refiere a "llenar") como el hecho de que sea aplicable a las cargas en movimiento e incluso a que puede pensarse en el campo de una única carga.

2. La justificación es explícita pero inaceptable: Incluye a respuestas que contienen referencias claras a justificaciones que no vinculan a la tesis con la conclusión.

Ejemplo para el tema 1:

Respuesta alumno: La fuerza se produce en la interacción entre dos cuerpos dentro de un campo eléctrico. La noción de campo nos determina si esos cuerpos son positivos o negativos en carga o si se encuentran en estado neutro, en cambio la noción de fuerza indica la interacción entre esas cargas y la distancia entre ambas

- Ejemplo para el tema 2:

Respuesta alumno: La importancia de este para describir el comportamiento de las cargas eléctricas es que, en las cargas eléctricas las cargas con distinto signo se atraen y las que tienen igual signo se repelen.

El estudiante presenta una justificación para dar cuenta de la importancia del modelo de campo que no es correcta aunque haga uso de una proposición verdadera tal como en las cargas eléctricas las cargas con distinto signo se atraen y las que tienen igual signo se repelen. La manifestación de atracción o de repulsión 
de las cargas es independiente del modelo que se emplee para describir la interacción.

Un caso particular de justificación explícita e inaceptable es el de la justificación circular: Incluye a las respuestas en las cuales para justificar el argumento se recurre a proposiciones que no agregan nada a lo que expresa el enunciado de la pregunta.

- Hay un único ejemplo de este tipo de justificación, para una respuesta correspondiente al tema 2 :

Respuesta alumno: Es bueno introducir la noción de campo para entender el comportamiento de una partícula cargada. Hay mucha relación entre estos.

La respuesta parece querer transmitir que la introducción de la noción de campo es importante sólo porque es bueno introducirla.

3. La justificación es implícita: en este caso se agrupan las repuestas que, aunque suelen presentar ambigüedades de redacción, permiten inferir posibles justificaciones .

- Algunos ejemplos:

Respuesta alumno: Cuando se habla de campo eléctrico se hace referencia a una condición que se genera en el espacio y que está asociado a las cargas. El campo eléctrico también va a ser asociado a una carga sola sin necesidad de que exista una segunda. En cambio, cuando se habla de fuerza estamos teniendo en cuenta que en un punto A se aplica una carga y que esta tiene asociado un campo el cual genera una condición que va a modificar el espacio por lo cual al aplicar una segunda carga en otro punto de ese campo se va a experimentar una fuerza.

Del enunciado anterior podría inferirse que en el caso de la fuerza hay necesidad de presencia de dos cargas. Hay dificultades para expresar que la fuerza se manifiesta sobre la segunda carga.

Respuesta alumno: La importancia es saber que hay otra particula cargada que lo afecta y como no están en contacto lo afecta mediante el campo eléctrico.

\footnotetext{
${ }^{6}$ Siendo que es posible realizar más de una inferencia cuando el texto es ambiguo, se opta por no discriminar entre aceptables y no aceptables.
} 
El estudiante incorpora el hecho de que la interacción resulta mediada por el campo. Sin embargo, no se especifica que ese campo incorpore a la noción de fuerza que es la responsable de afectar a una partícula cargada.

A modo de síntesis, se presenta la siguiente tabla con el único fin de comparar cualitativamente el análisis realizado para las preguntas de los dos temas.

\begin{tabular}{|c|c|l|l|l|l|l|}
\hline $\begin{array}{l}\text { Pre- } \\
\text { gunta } \\
\text { del }\end{array}$ & \multicolumn{2}{|c|}{$\begin{array}{l}\text { Tipo de concordan- } \\
\text { cia estructural }\end{array}$} & \multicolumn{4}{|c|}{ Tipo de justificación según el referente } \\
tema & Coherente & $\begin{array}{l}\text { No } \\
\text { cohe- } \\
\text { rente }\end{array}$ & $\begin{array}{l}\text { Explícita } \\
\text { y acepta- } \\
\text { ble }\end{array}$ & $\begin{array}{l}\text { Explícita } \\
\text { y no nar } \\
\text { aceptable }\end{array}$ & Implícita & $\begin{array}{l}\text { No hay } \\
\text { justifica- } \\
\text { ción }\end{array}$ \\
\hline 1 & 9 & 4 & 5 & 4 & 3 & 1 \\
\hline 2 & 8 & 2 & 2 & 6 & 2 & \\
\hline
\end{tabular}

Fig. 5 - Comparación de los patrones estructural y temático para las respuestas a las preguntas 1 y 2 .

La Fig. 5 presenta la comparación entre los tipos de justificación y, consecuentemente los patrones estructural y temático según las respuestas analizadas. Para cualquiera de las dos preguntas formuladas se advierte un número importante de respuestas que alcanzan la coherencia estructural. Es decir, la mayoría de los estudiantes es capaz de establecer una estructura argumentativa que vincule, coherentemente, la tesis, la conclusión y la justificación. Cuando esto no es posible, se dan los casos de justificaciones implícitas o simplemente no es posible, a partir de la repuesta ofrecida, reconocer a la justificación. Concretamente, los 4 casos que en la pregunta del tema 1 no son estructuralmente coherentes son los mismos cuya justificación no aparece o es implícita. De igual modo, los dos casos de justificación implícita del tema 2 se corresponden con la columna de concordancia no coherente.

Un aspecto importante de destacar es el hecho de la notable diferencia entre respuestas de justificación explícita y aceptable para los dos temas. Mientras que en el caso del tema 1 es mayoritario, el fenómeno se invierte cuando se analiza la pregunta del tema 2. Una posible explicación para esto podría estar en el tipo de cuestión formulada. El primer caso demanda la identificación de diferencias, en el 
segundo se solicita avalar la importancia de introducir una noción. Es decir, los estudiantes deberían dar razones que revaloricen a la incorporación de la noción de campo. Si bien, una manera relativamente, simple es la de reconocer que viene a "llenar" el "vacío" de la acción a distancia, es habitual que los estudiantes busquen destacar la importancia a partir de la funcionalidad que algo puede desenvolver. Es evidente que hay dificultades en la comprensión de que cada punto del espacio es, cuando se admite al campo, portador de una posible fuerza.

La demanda cognitiva de establecer diferencias presenta de manera clara cuál es el problema que se plantea en la pregunta, en cambio, un enunciado tan amplio como el de hablar sobre la importancia de la incorporación de un concepto parecería que, para la población participante, invita a enunciar una variedad de principios cuyo fundamento es, en algunos casos, valorativo pero ajeno a las razones científicas. En resumen, el tipo de pregunta del tema 2 promueve diversidad de opiniones más que de razones científicamente aceptables.

\section{Implicancias para la enseñanza de la Física}

Las principales implicancias para la enseñanza de la Física derivan de preguntarse ¿qué nos dicen los resultados anteriores acerca del proceso de aprendizaje de los estudiantes?

La primera aproximación para esta respuesta requiere definir un referente desde el cual poder establecer comparaciones. Siguiendo con la lectura de la tabla comparativa incluida bajo el título RESULTADOS, está claro que el ideal de aprendizaje está en la columna Justificación explícita y aceptable, asumiendo que las respuestas que aquí se concentran tienen, también, una Concordancia estructural coherente. En esos casos se estarían reconociendo respuestas que son tan aceptables desde el punto de vista del patrón estructural como desde el patrón temático.

Los resultados ponen de manifiesto que, entre las respuestas analizadas, es posible que la relación entre tesis, justificación y conclusión responda a los modelos de un texto argumentativo (es coherente estructuralmente), aunque el contenido de la justificación no sea científicamente aceptable (Justificación explícita y no aceptable).

Una cuestión interesante para discutir es qué decisiones tomar, como docente, al momento de la evaluación de respuestas de justificación implícita. ¿Qué

\footnotetext{
${ }^{7}$ Las respuestas analizadas son parte de una evaluación integradora.
} 
hacer frente a inferencias en apariencia aceptables, desde el punto de vista disciplinar, con estructuras que no son las de un texto argumentativo?

En la mayoría de los casos que esto ocurre, es posible reconocer alguna forma de falacia, es decir, que se viola alguna de las reglas de una buena argumentación.

Particularmente, asociado a los fines de evaluar las respuestas antes analizadas se reconocen violaciones a las siguientes tres reglas:

Regla 4: Los argumentos que defienden a una tesis deben estar relacionados con ella y no con otra tesis.

Ejemplo:

La importancia de la noción de campo eléctrico para describir el comportamiento de una partícula cargada es que el campo eléctrico sirve para saber qué signo poseen las cargas según las reacciones o qué ocurre en las mismas, los movimientos.

En este caso se usan argumentos irrelevantes o no pertinentes, ya que el signo de las cargas no tiene que ver con la presencia o ausencia de campo, es decir que la regla 4 no se está cumpliendo y en ese sentido se comete una falacia.

Regla 7: Una tesis puede considerarse defendida de forma adecuada si la defensa se desarrolla con el uso de argumentos que reflejen y respeten la praxis y el esquema argumentativo comúnmente aceptados.

Ejemplo:

La importancia es saber que hay otra partícula cargada que lo afecta y como no están en contacto lo afecta mediante el campo.

En este caso el argumento es una paráfrasis de la tesis citada, la justificación no agrega nada al enunciado de la pregunta y por tanto, la tesis no está defendida.

Regla 10: La formulación de las tesis, de las posiciones recíprocas y de los argumentos debe ser lo más clara y comprensible posible.

Ejemplo:

Básicamente se puede determinar que la noción de campo puede ser comprendida como parte de la fuerza eléctrica, es decir, que se encuentra contenido dentro de esta última. De este modo, como se presenta en la fórmula

$F=E / q_{p}$ la fuerza incluye, además del campo una carga de prueba que verifica la existencia de ese campo y el efecto que sufre como consecuencia de éste, determinando asi una interacción. 
La argumentación propuesta es ambigua y poco clara, parece querer traducir aquello que expresa la fórmula.

Ejemplos como los anteriores, que evidencian incompatibilidad entre la cuestión formulada y la respuesta ofrecida son muestras de aquello que no refleja un texto argumentativo sea por su estructura o sea porque se trata de una falacia. En ocasiones, es difícil reconocer que, aunque pudieran enunciarse expresiones correctas, desde el punto de vista científico no son ésas las expresiones que justifican lo que se ha consultado.

Situaciones como las expuestas, llevan a reconsiderar que la representación del espacio-problema que construye un estudiante difícilmente coincide con aquella que ha construido el docente (RICHARD, 1990). Esto pone de manifiesto la necesidad de acompañar a los estudiantes en ese camino de elaboración del espacio-problema (Newell y Simon, citado por Richard, op. cit.). En situaciones semánticamente ricas como las que aquí se han presentado, no es sencillo reconocer cuál es la situación de partida y cuál la posible vía de acceso a una llegada. Son situaciones que requieren capacidad para identificar aspectos singulares, interesantes, notables que permitan construir diferencias (pregunta del tema 1) y reconocer factores salientes en la evolución del conocimiento científico como es la incorporación de nuevos modelos explicativos de un determinado fenómeno (pregunta del tema 2).

No hay una estrategia universal para desenvolver procesos argumentativos. Los procesos argumentativos son netamente dependientes del contexto en que se encuentran insertos. Para promover la conceptualización de un cierto tema y para desarrollar un proceso argumentativo en relación a ese mismo tema, la situación que el docente proporcione a modo de "disparadora" es decisiva de lo que se desencadene a posteriori. En este sentido se enfatiza en la necesidad de profundizar sobre los alcances que plantea cada una de las situaciones que proponemos a los estudiantes. Entre otros aspectos a considerar se pueden destacar los siguientes:

- elaborar situaciones anticipando qué es lo que se espera construir con ellas;

- reconocer qué posibles obstáculos (conceptuales, de lenguaje, etc.) introduce la situación planteada;

- anticipar posibles respuestas de los alumnos para la situación propuesta;

- planear posibles intervenciones del docente para colaborar en el proceso de conceptualización;

- anticipar condiciones a partir de las cuales la situación propuesta se considerará respondida de manera aceptable. 
Los docentes necesitamos aprender mucho más acerca de discursos argumentativos y las implicancias que los mismos tienen en la formación del pensamiento crítico. Más aún, necesitamos decidir criterios de validación de los discursos de nuestros estudiantes para así poder garantizar un aprendizaje significativo.

\section{Referencias bibliográficas}

BERKSON, W. Las teorías de los campos de fuerza. Madrid: Alianza Editorial, 1981.

DÍAZ BARRIGA, F. Cognición situada y estrategias para el aprendizaje significativo. Revista electrónica de investigación educativa, v. 5, n. 2, 2003. Disponible en: $<$ http://redie.ens.uabc.mx/vol5no2/contenido-arceo.html $>$. Acceso en: 05 feb. 2007.

DRIVER, R.; ASOKO, H.; LEACH, J.; MORTIMER, E.; SCOTT, P. Constructing scientific knowledge in the classroom. Educational Researcher, v. 23, n. 7, p. 512, 1994.

JIMÉNEZ ALEXANDRE, P.; DÍAZ DE BUSTAMANTE J. Discurso de aula y argumentación en la clase de ciencias: cuestiones teóricas y metodológicas. Enseñanza de las Ciencias, v. 21, n. 3, p. 359-370, 2003.

LAKOFF, G. Women, fire and dangerous things. Chicago: The University of Chicago Press, 1987.

LEITAO, S. Argumentação como processo de construção do conhecimento. In: ENCONTRO INTERNACIONAL LINGUAGEM, CULTURA E COGNIÇÃO. REFLEXOES PARA O ENSINO, II, 2003, Belo Horizonte. Anais... (CD-ROM)

LEMKE, J. Talking science: language, learning and values. New Jersey: Ablex Publishing Corporation, 1990.

LEMKE, J. Aprender a hablar ciencia. Lenguaje, aprendizaje y valores. Barcelona: Paidós, 1997.

LERNER, D. Capacitación en servicio y cambio en la propuesta didáctica vigente. Lectura y vida, Año 15, n. 3 p. 33-47, 1994. 
LO CASCIO, V. Gramática de la argumentación. Madrid: Alianza Universidad, 1998.

MATURANA, H. La realidad ¿objetiva o construida? Fundamentos biológicos de la realidad. Chile: Editorial Anthropos/Itesa/U. Iberoamericana, 1995.

MORTIMER, E. Linguagem e formaçào de conceitos no ensino de Cièncias. Belo Horizonte: Editora UFMG, 2000.

NOVAK, J. Learning, creating, and using knowledge. Concept maps as facilitative tools in schools and corporations. N Jersey: Lawrence Erlbaum and Associates, 1998.

PÉREZ RIFO, M.; VEGA ALVARADO, O. Técnicas argumentativas. Chile: Ediciones Universidad Católica de Chile, 2003.

PERINAT, A. Prolegómenos para una teoría del símbolo y del signo. Cognitiva, v. 7, n. 2, p. 185-204, 1995.

PLANTÍN Pensar el debate. Revista signos, Valparaíso, v. 37, n. 55, 2004. Disponible em: < http://www.scielo.cl/>. Acceso em: jun. 2005.

POZO, J.; GÓMEZ CRESPO, M. Aprender y enseñar ciencia. Del conocimiento cotidiano al conocimiento científico. Madrid: Ediciones Morata, 1998.

RICHARD, J. F. Les activités mentales. París: A. Colin, 1990.

SÁNCHEZ ILABACA, J. Bases constructivistas para la integración de TICs. Revista enfoques educacionales, v. 6, n. 1, 2004. Disponible en:

$<$ http://www.facso.uchile.cl/publicaciones/enfoques/08/Sanchez Ilabaca.pdf $>$.

Acceso en: feb. 2006.

SARDÁ, J.; SANMARTÍ, N. Enseñar a argumentar científicamente: un reto de las clases de ciencias. Enseñanza de las Ciencias, v. 18, n. 3, p. 405-422, 2000.

SILVESTRI, A. El aprendizaje textual en la adolescencia: implicaciones cognitivas. In: JORNADAS EL LENGUAJE Y SUS ALTERACIONES, 1, 1997, Buenos Aires. Actas... Disponible en:

$<$ http://www.geocities.com/estudiscurso/silvestri1.html $>$. Acceso en: feb. 2006. 
STIPCICH, S. Invariantes operacionales en relación con la noción de campo eléctrico. In: SIMPOSIO DE INVESTIGADORES EN EDUCACIÓN EN FÍSICA, VIII, 2006, Gualeguaychú. Memorias... (CD-ROM)

SUTTON, C. Ideas sobre la ciencia e ideas sobre el lenguaje. Alambique, v. IV, n. 12, p. 8-32, 1997.

TOULMIN, S. The Uses of argument. Cambridge: Cambridge University Press, 1958.

TOULMIN, S. Regreso a la razón. El debate entre la racionalidad y la experiencia y la práctica personales en el mundo contemporáneo. Barcelona: Ediciones Península, 2003.

VAN EEMEREN, F.; GROOTENDORST, R. Argumentación, comunicación y falacias. Una perspectiva pragma-dialéctica. Chile: Ediciones Universidad Católica de Chile, 2002.

VIGOTSKY, L. Pensamiento y lenguaje. Buenos Aires: La Pléyade, 1963.

WERTSCH, J. Voces de la mente. Un enfoque sociocultural para el estudio de la acción mediada. España: Visor, 1993. 